1 Hacettepe Journal of Mathematics and Statistics

$\bigcap$ Volume $44(4)$ (2015), $901-908$

\title{
The frobenius problem for some numerical semigroups with embedding dimension equal to three
}

\author{
Aureliano M. Robles-Pérez* ${ }^{*}$ and José Carlos Rosales* ${ }^{*}$
}

\begin{abstract}
If $S$ is a numerical semigroup with embedding dimension equal to three whose minimal generators are pairwise relatively prime numbers, then $S=\langle a, b, c b-d a\rangle$ with $a, b, c, d$ positive integers such that $\operatorname{gcd}(a, b)=$ $\operatorname{gcd}(a, c)=\operatorname{gcd}(b, d)=1, c \in\{2, \ldots, a-1\}$, and $a<b<c b-d a$. In this paper we give formulas, in terms of $a, b, c, d$, for the genus, the Frobenius number, and the set of pseudo-Frobenius numbers of $\langle a, b, c b-d a\rangle$ in the case in which the interval $\left[\frac{a}{c}, \frac{b}{d}\right]$ contains some integer.
\end{abstract}

2000 AMS Classification: 11D07, 20M14.

Keywords: Frobenius problem, numerical semigroups, Apéry set.

Received 03/11/2012 : Accepted 27/03/2014 Doi : 10.15672 /HJMS.2015449432

\section{Introduction}

Let $\mathbb{Z}$ and $\mathbb{N}$ be the set of integers and the set of nonnegative integers, respectively. A numerical semigroup is a subset $S$ of $\mathbb{N}$ such that it is closed under addition, $0 \in S$, and $\mathbb{N} \backslash S$ is finite. The elements of $\mathbb{N} \backslash S$ are the gaps of $S$, and the cardinality of such set is called the genus of $S$, denoted by $\mathrm{g}(S)$. The Frobenius number of $S$ is the largest integer that does not belong to $S$ and it is denoted by $\mathrm{F}(S)$.

If $A \subseteq \mathbb{N}$ is a nonempty set, we denote by $\langle A\rangle$ the submonoid of $(\mathbb{N},+)$ generated by $A$, that is,

$$
\langle A\rangle=\left\{\lambda_{1} a_{1}+\ldots+\lambda_{n} a_{n} \mid n \in \mathbb{N} \backslash\{0\}, a_{1}, \ldots, a_{n} \in A, \text { and } \lambda_{1}, \ldots, \lambda_{n} \in \mathbb{N}\right\} .
$$

${ }^{*}$ Both of the authors are supported by FQM-343 (Junta de Andalucía), MTM2010-15595 (MICINN, Spain), and FEDER funds. The second author is also partially supported by Junta de Andalucía/Feder grant number FQM-5849.

${ }^{\dagger}$ Departamento de Matemática Aplicada, Universidad de Granada, 18071-Granada, Spain.

Email: arobles@ugr.es

${ }^{\ddagger}$ Departamento de Álgebra, Universidad de Granada, 18071-Granada, Spain.

Email: jrosales@ugr.es 
In [16] it is proved that $\langle A\rangle$ is a numerical semigroup if and only if $\operatorname{gcd}(A)=1$, where gcd means greatest common divisor.

It is well known (see [16]) that every numerical semigroup $S$ is finitely generated, that is, there exists a finite subset $G \subseteq S$ such that $S=\langle G\rangle$. In addition, if no proper subset of $G$ generates $S$, then we say that $G$ is a minimal system of generators of $S$. In [16] it is proved that every numerical semigroup admits a unique minimal system of generators. The cardinality of such a set is known as the embedding dimension of $S$, denoted by e $(S)$.

The Frobenius problem (see [8]) consists of finding formulas that allow us to compute, in terms of the minimal system of generators of a numerical semigroup, the Frobenius number and the genus of such a numerical semigroup. This problem was solved by Sylvester and Curran Sharp (see [18, 19, 20]) when the embedding dimension is equal to two. In fact, if $S$ is a numerical semigroup with minimal system of generators $\left\{n_{1}, n_{2}\right\}$, then $\mathrm{F}(S)=n_{1} n_{2}-n_{1}-n_{2}$ and $\mathrm{g}(S)=\frac{\left(n_{1}-1\right)\left(n_{2}-1\right)}{2}$.

At present, the Frobenius problem is open for the case of embedding dimension equal to three. To be precise, Curtis proved in [2] that it is impossible to find a polynomial formula that solves the problem of Frobenius number. On the other hand, algorithms that compute Frobenius number, quasi-formulas, and upper bounds for such number are the topic of several contributions (see $[4,8,9,11,12])$. In addition, the authors showed in [10] that, if the multiplicity of $S$ is fixed, then it is possible to give explicit formulas for the Frobenius number. In this paper, our purpose is to give simple formulas in a particular but extensive case (in the line of [7] and some results collected in [8]).

If $\left\{n_{1}, n_{2}, n_{3}\right\}$ is the minimal system of generators of a numerical semigroup $S$ and $d=\operatorname{gcd}\left(n_{1}, n_{2}\right)$, then we have (see $\left.[6,11]\right)$ that $\mathrm{F}(S)=d \mathrm{~F}\left(\left\langle\frac{n_{1}}{d}, \frac{n_{2}}{d}, n_{3}\right\rangle\right)+(d-1) n_{3}$ and $\mathrm{g}(S)=d \mathrm{~g}\left(\left\langle\frac{n_{1}}{d}, \frac{n_{2}}{d}, n_{3}\right\rangle\right)+\frac{(d-1)\left(n_{3}-1\right)}{2}$. Therefore, in order to solve the Frobenius problem for numerical semigroups with embedding dimension equal to three, we focus our attention on numerical semigroups whose three minimal generators are pairwise relatively prime numbers.

If $S$ is a numerical semigroup and $m \in S \backslash\{0\}$, then the Apéry set of $m$ in $S$ (see [1]) is $\operatorname{Ap}(S, m)=\{s \in S \mid s-m \notin S\}$. Obviously, $\operatorname{Ap}(S, m)=\{w(0)=0, w(1), \ldots, w(m-1)\}$, where $w(i)$ is the least element of $S$ congruent with $i$ modulo $m$, for all $i \in\{0, \ldots, m-1\}$. It is clear that $\mathrm{F}(S)=\max \{\operatorname{Ap}(S, m)\}-m$, and a formula for $\mathrm{g}(S)$ in terms of $\operatorname{Ap}(S, m)$ is given in [17] (see Lemma 3.1).

Following the notation introduced in [14], we say that $x \in \mathbb{Z} \backslash S$ is a pseudo-Frobenius number of $S$ if $x+s \in S$ for all $s \in S \backslash\{0\}$. We will denote by $\operatorname{PF}(S)$ the set of pseudoFrobenius numbers of $S$, and its cardinality is the type of $S$, denoted by $\operatorname{t}(S)$. From the definition it follows that $\mathrm{F}(S)=\max \{\mathrm{PF}(S)\}$.

In [15] it is shown that, if $S$ is a numerical semigroup with embedding dimension equal to three whose three minimal generators are pairwise relatively prime numbers, it is possible to describe $S$ in function of six positive integers $r_{12}, r_{13}, r_{21}, r_{23}, r_{31}, r_{32}$. Then, it is possible to give formulas for $\mathrm{F}(S), \mathrm{g}(S)$, and $\mathrm{PF}(S)$ in terms of such parameters. In this paper we will show that we can reduce the number of parameters to four $(a, b, c, d)$ and we will give the formulas for $\mathrm{F}(S), \mathrm{g}(S)$, and $\operatorname{PF}(S)$ in the case in which $\left[\frac{a}{c}, \frac{b}{d}\right] \cap \mathbb{N} \neq \emptyset$. We left as an open problem the case $\left[\frac{a}{c}, \frac{b}{d}\right] \cap \mathbb{N}=\emptyset$.

We summarize the content of this paper. Let us denote

$$
\begin{aligned}
\mathcal{F}=\{ & \langle a, b, c b-d a\rangle \mid a, b, c, d \in \mathbb{N} \backslash\{0\}, \operatorname{gcd}(a, b)=\operatorname{gcd}(a, c)=\operatorname{gcd}(b, d)=1, \\
& 2 \leq c \leq a-1, \text { and } a<b<c b-d a\} .
\end{aligned}
$$

First of all, we observe that $\mathcal{F}$ is the set of all numerical semigroups with embedding dimension equal to three whose minimal generators are pairwise relatively prime numbers. Let us consider $S=\langle a, b, c b-d a\rangle \in \mathcal{F}$. The main result in Section 2 is Theorem 2.8, 
where we give $\operatorname{Ap}(S, a)$ in an explicit way when $\left[\frac{a}{c}, \frac{b}{d}\right] \cap \mathbb{N} \neq \emptyset$. As a consequence of this result, in Section 3, we give the formulas for $\mathrm{F}(S), \mathrm{g}(S)$, and $\mathrm{PF}(S)$ in the above mentioned case.

\section{The Apéry set}

Our purpose in this section is to prove Theorem 2.8, where we will show explicitly an Apéry set for a particular family of numerical semigroups with embedding dimension three. It is well known that the Apéry set allows us to solve easily the Frobenius problem, as well as simplify many questions about numerical semigroups, such as the membership problem (that is, determine if a positive integer belongs to a numerical semigroup).

First we need to introduce some results. The following lemma has an immediate proof (see [16, Lemma 2.6]).

2.1. Lemma. Let $S$ be a numerical semigroup and $m \in S \backslash\{0\}$. Then, for every $x \in \mathbb{Z}$, there exist a unique $(\lambda, w) \in \mathbb{Z} \times \operatorname{Ap}(S, m)$ such that $x=\lambda m+w$. Moreover, $x \in S$ if and only if $\lambda \in \mathbb{N}$.

Let $p, q$ be two integers such that $q \neq 0$. We denote by $\left\lfloor\frac{p}{q}\right\rfloor$ and $p \bmod q$ the quotient and the remainder of the integer division of $p$ by $q$, respectively. The next result follows from [13, Lemma 3.3].

2.2. Lemma. Let $m$ be a positive integer. Let $\{X(0)=0, X(1), \ldots, X(m-1)\}$ be a subset of $\mathbb{N}$ such that $X(i) \bmod m=i$, for all $i \in\{0,1, \ldots, m-1\}$. Let $S=$ $\langle m, X(1), \ldots, X(m-1)\rangle$. Then $\operatorname{Ap}(S, m)=\{X(0), X(1), \ldots, X(m-1)\}$ if and only if $X(i)+X(j) \geq X((i+j) \bmod m)$, for all $i, j \in\{1, \ldots, m-1\}$.

The following lemma is well known (see, for instance, [17]).

2.3. Lemma. Let $n_{1}, n_{2}$ be two positive integers such that $\operatorname{gcd}\left(n_{1}, n_{2}\right)=1$. Let $S=$ $\left\langle n_{1}, n_{2}\right\rangle$. Then $\operatorname{Ap}\left(S, n_{1}\right)=\left\{0, n_{2}, 2 n_{2}, \ldots,\left(n_{1}-1\right) n_{2}\right\}$.

2.4. Lemma. Let $S$ be a numerical semigroup with minimal system of generators given by $\left\{n_{1}, n_{2}, n_{3}\right\}$. If $\operatorname{gcd}\left(n_{1}, n_{2}\right)=1$, then there exist two unique numbers $k \in\left\{2, \ldots, n_{1}-1\right\}$ and $t \in\left\{1, \ldots, n_{2}-1\right\}$ such that $n_{3}=k n_{2}-t n_{1}$.

Proof. Since $n_{3} \notin\left\langle n_{1}, n_{2}\right\rangle$, by Lemmas 2.1 and 2.3, we deduce that there exist unique numbers $k \in\left\{0,1, \ldots, n_{1}-1\right\}$ and $t \in \mathbb{N} \backslash\{0\}$ such that $n_{3}=k n_{2}-t n_{1}$. Since $n_{3}>0$, it is obvious that $t \in\left\{1, \ldots, n_{2}-1\right\}$. In order to finish the proof we have to see that $k \notin\{0,1\}$. If $k=0$, then $n_{3}=-t n_{1}$, which is a contradiction to the positiveness of $n_{3}$. If $k=1$, then $n_{2}=n_{3}+t n_{1} \in\left\langle n_{1}, n_{3}\right\rangle$, which is a contradiction because $\left\{n_{1}, n_{2}, n_{3}\right\}$ is a minimal system of generators.

2.5. Remark. By using Euclidean algorithm, we have that, if $\operatorname{gcd}\left\{n_{1}, n_{2}\right\}=1$, then there exist two positive integers $u, v$ such that $u n_{2}-v n_{1}=1$. Thus, $n_{3}=\left(u n_{3}\right) n_{2}-$ $\left(v n_{3}\right) n_{1}=\left(\left\lfloor\frac{u n_{3}}{n_{1}}\right\rfloor n_{1}+\left(u n_{3}\right) \bmod n_{1}\right) n_{2}-\left(v n_{3}\right) n_{1}$. Therefore $n_{3}=\left(\left(u n_{3}\right) \bmod n_{1}\right) n_{2}-$ $\left(v n_{3}-\left\lfloor\frac{u n_{3}}{n_{1}}\right\rfloor n_{2}\right) n_{1}$. We conclude that, in Lemma $2.4, k=\left(u n_{3}\right) \bmod n_{1}$ and $t=$ $v n_{3}-\left\lfloor\frac{u n_{3}}{n_{1}}\right\rfloor n_{2}$.

2.6. Remark. Let $S$ be a numerical semigroup with minimal system of generators $\left\{n_{1}, n_{2}, n_{3}\right\}$. If $n_{1}, n_{2}, n_{3}$ are pairwise relatively prime numbers and $n_{1}<n_{2}<n_{3}$, taking $a=n_{1}, b=n_{2}, c=k, d=t$, and $c b-d a=k n_{2}-t n_{1}=n_{3}$, we deduce from Lemma 2.4 that $S \in \mathcal{F}$. On the other hand, it is easy to see that any element of $\mathcal{F}$ is a numerical semigroup with embedding dimension equal to three whose minimal generators are pairwise relatively prime numbers. 
2.7. Lemma. Let $a, b, c, d$ be four positive integers such that $\operatorname{gcd}(a, b)=1$ and $c b-d a \geq$ $d((-a) \bmod c)$. Let $S=\left\langle\left\{\alpha b-\left\lfloor\frac{\alpha}{c}\right\rfloor d a \mid \alpha \in\{0, \ldots, a-1\}\right\} \cup\{a\}\right\rangle$. Then

$$
\operatorname{Ap}(S, a)=\left\{\alpha b-\left\lfloor\frac{\alpha}{c}\right\rfloor d a \mid \alpha \in\{0, \ldots, a-1\}\right\} .
$$

Proof. Since $c b-d a \geq 0$, it follows that $\alpha b-\left\lfloor\frac{\alpha}{c}\right\rfloor d a \in \mathbb{N}$, for all $\alpha \in\{0, \ldots, a-1\}$. Moreover, $\left\{\alpha b-\left\lfloor\frac{\alpha}{c}\right\rfloor d a \mid \alpha \in\{0, \ldots, a-1\}\right\}$ is a subset of $\mathbb{N}$ such that it has the form $\{X(0)=0, X(1), \ldots, X(a-1)\}$ with $X(i) \bmod a=i$, for all $i \in\{0,1, \ldots, a-1\}$. Then, by using Lemma 2.2 , we conclude the proof if we show both of the next two statements.

(1) If $\alpha, \beta \in\{1, \ldots, a-1\}$ and $\alpha+\beta \leq a-1$, then

$$
\alpha b-\left\lfloor\frac{\alpha}{c}\right\rfloor d a+\beta b-\left\lfloor\frac{\beta}{c}\right\rfloor d a \geq(\alpha+\beta) b-\left\lfloor\frac{\alpha+\beta}{c}\right\rfloor d a .
$$

(2) If $\alpha, \beta \in\{1, \ldots, a-1\}$ and $\alpha+\beta \geq a$, then

$$
\alpha b-\left\lfloor\frac{\alpha}{c}\right\rfloor d a+\beta b-\left\lfloor\frac{\beta}{c}\right\rfloor d a \geq(\alpha+\beta-a) b-\left\lfloor\frac{\alpha+\beta-a}{c}\right\rfloor d a .
$$

The first one follows from the inequality $\left\lfloor\frac{\alpha+\beta}{c}\right\rfloor \geq\left\lfloor\frac{\alpha}{c}\right\rfloor+\left\lfloor\frac{\beta}{c}\right\rfloor$. The second one is equivalent to $\frac{b}{d} \geq\left\lfloor\frac{\alpha}{c}\right\rfloor+\left\lfloor\frac{\beta}{c}\right\rfloor-\left\lfloor\frac{\alpha+\beta-a}{c}\right\rfloor$. Since $\left\lfloor\frac{x}{c}\right\rfloor c=x-x \bmod c$, for all $x \in \mathbb{N}$, if we multiply both sides of the inequality by $c$, then it suffices to prove that

$$
\frac{c b}{d} \geq \alpha-\alpha \bmod c+\beta-\beta \bmod c-(\alpha+\beta-a)+(\alpha+\beta-a) \bmod c,
$$

or equivalently, that

$$
\frac{c b}{d} \geq a+(\alpha+\beta-a) \bmod c-\alpha \bmod c-\beta \bmod c .
$$

Now, let us observe that $(\alpha+\beta-a) \bmod c \leq \alpha \bmod c+\beta \bmod c+(-a) \bmod c$. Since $c b-d a \geq d((-a) \bmod c)$, we conclude that

$$
\begin{aligned}
\frac{c b}{d} & \geq a+(-a) \bmod c \\
& =a+(-a) \bmod c+\alpha \bmod c+\beta \bmod c-\alpha \bmod c-\beta \bmod c \\
& \geq a+(\alpha+\beta-a) \bmod c-\alpha \bmod c-\beta \bmod c .
\end{aligned}
$$

Let $q$ be a rational number. As usual, we denote by $\lceil q\rceil$ the minimum of the set $\{z \in \mathbb{Z} \mid q \leq z\}$. At this point, we are in a position to prove the main result of this section.

2.8. Theorem. Let $S=\langle a, b, c b-d a\rangle \in \mathcal{F}$. If $\left[\frac{a}{c}, \frac{b}{d}\right] \cap \mathbb{N} \neq \emptyset$, then

$$
\operatorname{Ap}(S, a)=\left\{\alpha b-\left\lfloor\frac{\alpha}{c}\right\rfloor d a \mid \alpha \in\{0, \ldots, a-1\}\right\} .
$$

Proof. First of all, let us observe that, since $\left\lceil\frac{x}{c}\right\rceil c=x+(-x) \bmod c$, for all $x \in \mathbb{N}$, then

$$
c b-d a \geq d((-a) \bmod c) \Leftrightarrow c b-d a \geq d\left(\left\lceil\frac{a}{c}\right\rceil c-a\right) \Leftrightarrow \frac{b}{d} \geq\left\lceil\frac{a}{c}\right\rceil .
$$

Obviously, the last inequality is precisely the condition $\left[\frac{a}{c}, \frac{b}{d}\right] \cap \mathbb{N} \neq \emptyset$.

Now, from Lemma 2.7, if $\bar{S}=\left\langle\left\{\alpha b-\left\lfloor\frac{\alpha}{c}\right\rfloor d a \mid \alpha \in\{0, \ldots, a-1\}\right\} \cup\{a\}\right\rangle$, then we have that $\operatorname{Ap}(\bar{S}, a)=\left\{\alpha b-\left\lfloor\frac{\alpha}{c}\right\rfloor d a \mid \alpha \in\{0, \ldots, a-1\}\right\}$. Therefore, to finish the proof, it is enough to show that $S=\bar{S}$.

Since $c \geq 2$, then $\left\lfloor\frac{1}{c}\right\rfloor=0$ and $b=b-\left\lfloor\frac{1}{c}\right\rfloor d a \in \bar{S}$. Moreover, it is obvious that $a \in \bar{S}$ and $c b-d a=c b-\left\lfloor\frac{c}{c}\right\rfloor d a \in \bar{S}$. Therefore, $S=\langle a, b, c b-d a\rangle \subseteq \bar{S}$. For the other inclusion, let us take $x \in \bar{S}$. From Lemma 2.1, we deduce that there exist $\lambda \in \mathbb{N}$ and 
$\alpha \in\{0, \ldots, a-1\}$ such that $x=\lambda a+\left(\alpha b-\left\lfloor\frac{\alpha}{c}\right\rfloor d a\right)$. Since $x=\lambda a+\left(\left\lfloor\frac{\alpha}{c}\right\rfloor c+\alpha \bmod c\right) b-$ $\left\lfloor\frac{\alpha}{c}\right\rfloor d a=\lambda a+(\alpha \bmod c) b+\left\lfloor\frac{\alpha}{c}\right\rfloor(c b-d a)$, then $x \in\langle a, b, c b-d a\rangle=S$.

2.9. Remark. Let us observe that $\mathcal{F}$ contains infinitely many numerical semigroups (in fact, as it is pointed out in Remark 2.6, all numerical semigroups with embedding dimension equal to three whose minimal generators are pairwise relatively prime numbers) but not all of them satisfy the condition of Theorem 2.8 , that is, $\left[\frac{a}{c}, \frac{b}{d}\right]$ contains some integer. For example, the numerical semigroup $S=\langle 16,19,7 \times 19-7 \times 16\rangle=\langle 16,19,21\rangle$ does not satisfy that condition. Even more (see Remark 3.8 and Example 3.13), no possible combination of $a, b, c, d$ for this numerical semigroup satisfies the condition of Theorem 2.8.

2.10. Example. Let $S=\langle 5,7,3 \times 7-2 \times 5\rangle=\langle 5,7,11\rangle$. Since $\frac{7}{2} \geq\left\lceil\frac{5}{3}\right\rceil$, by Theorem 2.8 , we have that

$$
\operatorname{Ap}(S, 5)=\left\{\alpha \times 7-\left\lfloor\frac{\alpha}{3}\right\rfloor \times 2 \times 5 \mid \alpha \in\{0,1,2,3,4\}\right\}=\{0,7,14,11,18\} .
$$

\section{The genus and the pseudo-Frobenius numbers}

Along this section $S$ is a numerical semigroup which belongs to $\mathcal{F}$. Therefore, $S=$ $\langle a, b, c b-d a\rangle$ with $a, b, c, d$ positive integers such that $\operatorname{gcd}(a, b)=\operatorname{gcd}(a, c)=\operatorname{gcd}(b, d)=1$, $2 \leq c \leq a-1$, and $a<b<c b-d a$. Moreover, we suppose that $\frac{b}{d} \geq\left\lceil\frac{a}{c}\right\rceil$. Our purpose is to give formulas for $\mathrm{g}(S), \mathrm{PF}(S)$, and $\mathrm{F}(S)$.

The following result appears in [17].

3.1. Lemma. If $T$ is a numerical semigroup and $m \in T \backslash\{0\}$, then

$$
\mathrm{g}(T)=\left(\frac{1}{m} \sum_{w \in \operatorname{Ap}(T, m)} w\right)-\frac{m-1}{2} .
$$

Let us show a formula to compute $\mathrm{g}(S)$.

3.2. Proposition. Let $S$ be a numerical semigroup which satisfies the conditions stated at the beginning of this section. Then

$$
\mathrm{g}(S)=\frac{(b-1)(a-1)}{2}-d\left\lfloor\frac{a-1}{c}\right\rfloor\left(a-\frac{c}{2}\left(\left\lfloor\frac{a-1}{c}\right\rfloor+1\right)\right) .
$$

Proof. From Theorem 2.8 and Lemma 3.1, we have that

$$
\begin{aligned}
\mathrm{g}(S) & =\frac{1}{a} \sum_{\alpha=1}^{a-1}\left(\alpha b-\left\lfloor\frac{\alpha}{c}\right\rfloor d a\right)-\frac{a-1}{2}=\frac{1}{a}\left(b \sum_{\alpha=1}^{a-1} \alpha-d a \sum_{\alpha=1}^{a-1}\left\lfloor\frac{\alpha}{c}\right\rfloor\right)-\frac{a-1}{2} \\
& =\frac{1}{a}\left(b \frac{a(a-1)}{2}-d a \sum_{\alpha=1}^{a-1}\left\lfloor\frac{\alpha}{c}\right\rfloor\right)-\frac{a-1}{2}=\frac{(b-1)(a-1)}{2}-d \sum_{\alpha=1}^{a-1}\left\lfloor\frac{\alpha}{c}\right\rfloor .
\end{aligned}
$$

In order to finish the proof we need to show that

$$
\sum_{\alpha=1}^{a-1}\left\lfloor\frac{\alpha}{c}\right\rfloor=\left\lfloor\frac{a-1}{c}\right\rfloor\left(a-\frac{c}{2}\left(\left\lfloor\frac{a-1}{c}\right\rfloor+1\right)\right) .
$$


Let us observe that $\alpha \in\{i c, i c+1, \ldots, i c+(c-1)\}$ if and only if $\left\lfloor\frac{\alpha}{c}\right\rfloor=i$. Therefore,

$$
\begin{aligned}
\sum_{\alpha=1}^{a-1}\left\lfloor\frac{\alpha}{c}\right\rfloor & =\sum_{j=1}^{\left\lfloor\frac{a-1}{c}\right\rfloor-1}\left(\sum_{\alpha=0}^{c-1} j\right)+\sum_{\alpha=0}^{a-1-\left\lfloor\frac{a-1}{c}\right\rfloor c}\left\lfloor\frac{a-1}{c}\right\rfloor \\
& =c \sum_{j=1}^{\left\lfloor\frac{a-1}{c}\right\rfloor-1} j+\left\lfloor\frac{a-1}{c}\right\rfloor\left(a-\left\lfloor\frac{a-1}{c}\right\rfloor c\right) \\
& =\left\lfloor\frac{a-1}{c}\right\rfloor\left(\frac{c}{2}\left(\left\lfloor\frac{a-1}{c}\right\rfloor-1\right)+a-c\left\lfloor\frac{a-1}{c}\right\rfloor\right) .
\end{aligned}
$$

3.3. Example. Let $S=\langle 5,7,3 \times 7-2 \times 5\rangle=\langle 5,7,11\rangle$. Since $\frac{7}{2} \geq\left\lceil\frac{5}{3}\right\rceil$, applying Proposition 3.2 , we have that

$$
\mathrm{g}(S)=\frac{6 \times 4}{2}-2\left\lfloor\frac{4}{3}\right\rfloor\left(5-\frac{3}{2}\left(\left\lfloor\frac{4}{3}\right\rfloor+1\right)\right)=12-4=8 .
$$

In [3] it is shown that, if $T$ is a numerical semigroup with embedding dimension equal to three, then $\mathrm{t}(T) \in\{1,2\}$. Moreover, $t(T)=1$ if and only if $T$ is a symmetric numerical semigroup. In [5] it is proved that a numerical semigroup $T$ with embedding dimension equal to three is symmetric if and only if is a complete intersection numerical semigroup, and then the minimal generators of $T$ can not be pairwise relatively prime numbers. Therefore, if $S$ is a numerical semigroup such as at the beginning of this section, then $\mathrm{t}(S)=2$. We can give explicitly the elements of $\operatorname{PF}(S)$. But first we need a lemma.

Let $T$ be a numerical semigroup. We define in $T$ the partial order

$$
x \leq_{T} y \text { if } y-x \in T .
$$

If $A \subseteq T$, we denote by $\max _{\leq_{T}}\{A\}$ the set of maximals elements of $A$ with respect to the previous partial order. From [3, Proposition 7] we deduce the following result.

3.4. Lemma. Let $T$ be a numerical semigroup and $m \in T \backslash\{0\}$. If we set $\left\{w_{i 1}, \ldots, w_{i t}\right\}=$ $\max _{\leq_{T}}\{\operatorname{Ap}(T, m)\}$, then $\operatorname{PF}(T)=\left\{w_{i 1}-m, \ldots, w_{i t}-m\right\}$.

3.5. Proposition. Let $S$ be a numerical semigroup such as at the beginning of this section. Then

$$
\operatorname{PF}(S)=\left\{\left\lfloor\frac{a-1}{c}\right\rfloor(c b-d a)+d a-b-a,(a-1) b-\left\lfloor\frac{a-1}{c}\right\rfloor d a-a\right\} .
$$

Proof. From Theorem 2.8 we know that

$$
\operatorname{Ap}(S, a)=\left\{\alpha b-\left\lfloor\frac{\alpha}{c}\right\rfloor d a \mid \alpha \in\{0, \ldots, a-1\}\right\} .
$$

Since $\alpha b-\left\lfloor\frac{\alpha}{c}\right\rfloor d a=\left(\left\lfloor\frac{\alpha}{c}\right\rfloor c+\alpha \bmod c\right) b-\left\lfloor\frac{\alpha}{c}\right\rfloor d a=\left\lfloor\frac{\alpha}{c}\right\rfloor(c b-d a)+(\alpha \bmod c) b$ and $(a-1) \bmod c \neq c-1$ (in case of equality, we have $a \bmod c=0$, and then $c \mid a$, which is a contradiction with $\operatorname{gcd}(a, c)=1)$, we have that $\max _{\leq_{S}}\{\operatorname{Ap}(S, a)\}=$

$$
\left\{\left(\left\lfloor\frac{a-1}{c}\right\rfloor-1\right)(c b-d a)+(c-1) b,\left\lfloor\frac{a-1}{c}\right\rfloor(c b-d a)+((a-1) \bmod c) b\right\} .
$$

Having in mind that $(a-1) \bmod c=a-1-\left\lfloor\frac{a-1}{c}\right\rfloor c$, then

Therefore,

$$
\left\lfloor\frac{a-1}{c}\right\rfloor(c b-d a)+((a-1) \bmod c) b=-\left\lfloor\frac{a-1}{c}\right\rfloor d a+(a-1) b .
$$

$$
\max _{\leq_{S}}\{\operatorname{Ap}(S, a)\}=\left\{\left\lfloor\frac{a-1}{c}\right\rfloor(c b-d a)+d a-b,(a-1) b-\left\lfloor\frac{a-1}{c}\right\rfloor d a\right\} .
$$


From Lemma 3.4 we conclude the proof.

3.6. Example. Let $S=\langle 5,7,3 \times 7-2 \times 5\rangle=\langle 5,7,11\rangle$. Since $\frac{7}{2} \geq\left\lceil\frac{5}{3}\right\rceil$, applying Proposition 3.5, we have that $\operatorname{PF}(S)=\{9,13\}$.

3.7. Corollary. Let $S$ be a numerical semigroup such as at the beginning of this section. Then

$$
\mathrm{F}(S)= \begin{cases}(a-1) b-\left\lfloor\frac{a-1}{c}\right\rfloor d a-a, & \text { if } 1>\left\lfloor\frac{a-1}{c}\right\rfloor \frac{c}{a}+\frac{d}{b}, \\ \left\lfloor\frac{a-1}{c}\right\rfloor(c b-d a)+d a-b-a, & \text { in other case. }\end{cases}
$$

Proof. Since $\mathrm{F}(S)=\max \{\mathrm{PF}(S)\}$, then it is enough to apply Proposition 3.5 and note that $(a-1) b-\left\lfloor\frac{a-1}{c}\right\rfloor d a-a>\left\lfloor\frac{a-1}{c}\right\rfloor(c b-d a)+d a-b-a$ if and only if $1>\left\lfloor\frac{a-1}{c}\right\rfloor \frac{c}{a}+\frac{d}{b}$.

3.8. Remark. It is possible to improve the results of this paper. Indeed, we only need to impose the conditions $0<c b-d a$ and $\mathrm{e}(S)=3$ instead of the condition $a<b<c b-d a$. This way, if we consider the set

$$
\begin{aligned}
\mathcal{F}^{*}=\{ & \langle a, b, c b-d a\rangle \mid a, b, c, d \in \mathbb{N} \backslash\{0\}, \operatorname{gcd}(a, b)=\operatorname{gcd}(a, c)=\operatorname{gcd}(b, d)=1, \\
& 2 \leq c \leq a-1, c b-d a>0, \text { and } \mathrm{e}(\langle a, b, c b-d a\rangle)=3\},
\end{aligned}
$$

it is easy to see that $S=\langle a, b, c b-d a\rangle \in \mathcal{F}^{*}$ is a numerical semigroup with minimal system of generators whose elements are pairwise relatively prime numbers. Reciprocally, following the ideas of Remarks 2.5 and 2.6, if $S$ is a numerical semigroup with minimal system of generators $\left\{n_{1}, n_{2}, n_{3}\right\}$ and $n_{1}, n_{2}, n_{3}$ are pairwise relatively prime numbers, then $S \in \mathcal{F}^{*}$. Moreover, if we make some minor changes at the exposed reasonings in this section and the previous one, we get that Theorem 2.8, Propositions 3.2 and 3.5, and Corollary 3.7 remain true.

3.9. Remark. In fact, as sets, $\mathcal{F}=\mathcal{F}^{*}$. The difference between both of them is that, if $S$ is a numerical semigroup with embedding dimension three and minimal system of generators formed by pairwise relatively prime numbers, then $S$ has a unique representation in $\mathcal{F}$ and six representations in $\mathcal{F}^{*}$. On the other hand, we have that all the numerical semigroups in $\mathcal{F}$ has dimension three automatically, but in $\mathcal{F}^{*}$ we have to impose explicitly such a condition.

3.10. Example. Let $S=\langle 6,7,11\rangle$. We have that $(6,7,5,4)$ is the unique combination associated to $S$ in $\mathcal{F}$. The six combinations for $S$ in $\mathcal{F}^{*}$ are $(6,7,5,4),(6,11,5,8)$, $(7,6,3,1),(7,11,5,7),(11,6,3,1)$, and $(11,7,4,2)$.

3.11. Example. If we take the combination $(a, b, c, d)=(3,7,2,4)$, we have the numerical semigroup $S=\langle 3,7,2 \times 7-4 \times 3\rangle=\langle 3,7,2\rangle=\langle 2,3\rangle$. If we try to apply our results, we will have wrong answers.

3.12. Example. Let $S=\langle 6,7,11\rangle$. If we take $a=6, b=7, c=5$, and $d=4$, then $\frac{7}{4}<\left\lceil\frac{6}{5}\right\rceil$. Therefore, we can not apply the results. However, if we take $a=7, b=6$, $c=3$, and $d=1$, then $\frac{6}{1} \geq\left\lceil\frac{7}{3}\right\rceil$. In this case we have that,

- by Theorem 2.8 ,

$$
\begin{gathered}
\operatorname{Ap}(S, 7)=\left\{\alpha \times 6-\left\lfloor\frac{\alpha}{3}\right\rfloor \times 1 \times 7 \mid \alpha \in\{0,1, \ldots, 6\}\right\}= \\
\{0,6,12,11,17,23,22\}
\end{gathered}
$$

- by Proposition 3.2,

$$
\mathrm{g}(S)=\frac{5 \times 6}{2}-1\left\lfloor\frac{6}{3}\right\rfloor\left(7-\frac{3}{2}\left(\left\lfloor\frac{6}{3}\right\rfloor+1\right)\right)=15-5=10 ;
$$

- by Proposition 3.5, $\operatorname{PF}(S)=\{16,15\}$. 
3.13. Example. Let $S$ be the numerical semigroup generated by $\{16,19,21\}$. It is easy to check that there not exists a combination of $a, b, c, d$ (associated to $S$ ) such that the condition $\left[\frac{a}{c}, \frac{b}{d}\right] \cap \mathbb{N} \neq \emptyset$ is satisfied.

Let $S$ be a numerical semigroup. We denote by $\operatorname{m}(S)=\min (S \backslash\{0\})$, which it is called the multiplicity of $S$. We finish this paper with the following conjecture.

3.14. Conjecture. Let $S$ be a numerical semigroup such that $\mathrm{m}(S) \leq 15$. Then it is possible to find a combination of $a, b, c, d$ (associated to $S$ ) in such a way the condition $\left[\frac{a}{c}, \frac{b}{d}\right] \cap \mathbb{N} \neq \emptyset$ is satisfied.

Acknowledgements. The authors would like to thank the referees for their useful comments and suggestions that helped to improve this work.

\section{References}

[1] Apéry, R. Sur les branches superlinéaires des courbes algébriques, C. R. Acad. Sci. Paris 222, 1198-1200, 1946.

[2] Curtis, F. On formulas for the Frobenius number of a numerical semigroup, Math. Scand. 67, 190-192, 1990.

[3] Fröberg, R., Gottlieb, G. and Häggkvist, R. On numerical semigroups, Semigroup Forum 35, 63-83, 1987.

[4] Greenberg, H. Solution to a Diophantine equation for nonnegative integers, J. Algorithms 9, 343-353, 1988.

[5] Herzog, J. Generators and relations of abelian semigroups and semigroup rings, Manuscripta Math. 3, 175-193, 1970.

[6] Johnson, S. M. A linear Diophantine problem, Canad. J. Math. 12, 390-398, 1960.

[7] Marín, J. M., Ramírez Alfonsín, J. L. and Revuelta, M. P. On the Frobenius number of Fibonacci numerical semigroups, Integers 7, 7pp., 2007.

[8] Ramírez Alfonsín, J. L. The Diophantine Frobenius problem (Oxford Lecture Series in Mathematics and its Applications, 30, Oxford University Press, London, 2005).

[9] Ramírez Alfonsín, J. L. and Rødseth, Ø. J. Numerical semigroups: Apéry sets and Hilbert series, Semigroup Forum 79, 323-340, 2009.

[10] Robles-Pérez, A. M. and Rosales, J. C. The Frobenius problem for numerical semigroups with embedding dimension equal to three, Math. Comput. 81, 1609-1617, 2012.

[11] Rödseth, Ö. J. On a linear Diophantine problem of Frobenius, J. Reine Angew. Math. 301, 171-178, 1978.

[12] Rödseth, Ö. J. On a linear Diophantine problem of Frobenius II, J. Reine Angew. Math. 307/308, 431-440, 1979.

[13] Rosales, J. C. On numericals semigroups, Semigroup Forum 52, 307-318, 1996.

[14] Rosales, J. C. and Branco, M. B. Numerical semigroups that can be expressed as an intersection of symmetric numerical semigroups, J. Pure Appl. Algebra 171, 303-314, 2002.

[15] Rosales, J. C. and García-Sánchez, P. A. Numerical semigroups with embedding dimension three, Arch. Math. 83, 488-496, 2004.

[16] Rosales, J. C. and García-Sánchez, P. A. Numerical semigroups (Developments in Mathematics, vol. 20, Springer, New York, 2009).

[17] Selmer, E. S. On the linear Diophantine problem of Frobenius, J. Reine Angew. Math. 293/294, 1-17, 1977.

[18] Sylvester, J. J. On subvariants, i.e. semi-invariants to binary quantics of an unlimited order, Amer. J. Math. 5, 79-136, 1882.

[19] Sylvester, J. J. Problem 7382, The Educational Times, and Journal of the College of Preceptors, New Series, 36 (266), 177, 1883. Solution by W. J. Curran Sharp, ibid., 36 (271), 315, 1883. Republished as [20].

[20] Sylvester, J. J. Problem 7382, in W. J. C. Miller (Ed.), Mathematical questions, with their solutions, from "The Educational Times", vol. 41, page 21, Francis Hodgson, London, 1884 